Ebisu

Études japonaises

Études japonaises

48 automne-hiver 2012

Naissance d'une revue féministe au Japon : Seitō

(1911-1916)

\title{
Le premier débat public de Seitō : autour d'Une Maison de poupée
}

The First Public Debate of Seitō: Around A Doll's House

\author{
『青靱』の最初の公開討論—『人形の家』をめぐって一
}

\section{Christine Lévy}

\section{OpenEdition \\ Journals}

Édition électronique

URL : http://journals.openedition.org/ebisu/569

DOI : 10.4000/ebisu.569

ISSN : 2189-1893

Éditeur :

Institut français de recherche sur le Japon (UMIFRE 19 MAEE-CNRS), Maison franco-japonaise

Édition imprimée

Date de publication : 1 septembre 2012

Pagination : 29-58

ISSN : 1340-3656

Référence électronique

Christine Lévy, "Le premier débat public de Seitō : autour d'Une Maison de poupée », Ebisu [En ligne], 48 | automne-hiver 2012, mis en ligne le 21 mai 2014, consulté le 18 septembre 2019. URL : http:// journals.openedition.org/ebisu/569; DOI : 10.4000/ebisu.569 


\title{
Le premier débat public de Seitō Autour d'Une Maison de poupée
}

\author{
Christine LÉVY*
}

\section{L'entrée en scène des femmes}

En novembre 1911, la pièce du dramaturge norvégien Henrik Ibsen, Une Maison de poupée, fut présentée pour la première fois à Tokyo, au théâtre impérial inauguré au mois de mars de la même année. L'actrice Matsui Sumako 松井須磨子 (1886-1919) ${ }^{1}$ y était dirigée par le dramaturge Shimamura Hōgetsu 島村抱月 (1871-1918) ${ }^{2}$. Dans ce pays où les femmes avaient été exclues de la scène théâtrale depuis 1629 et ce officiellement jusqu'en $1891^{3}$, cette représentation marqua un double événement : d'une

* UMIFRE 19 MAEE-CNRS MFJ, université de Bordeaux 3.

1. Pour une étude du rôle joué par cette actrice dans la naissance du théâtre moderne au Japon et dans le féminisme, voir Kano (2001), en particulier la troisième partie.

2. Fondateur avec Tsubouchi Shōyō 坪内逍遥 de la Société des arts littéraires Bungei kyōkai 文藝協会, il devint l'amant de Matsui Sumako lors de la mise en scène d'Une Maison de poupée. Lorsque cette relation devint objet de scandale public, Tsubouchi exigea la démission de Matsui. Hōgetsu divorça, démissionna à la fois de l'association et de son poste de professeur pour fonder, en 1913, avec Matsui Sumako, le Théâtre des arts (Geijutsuza 芸術座) où fleurit le théâtre nouveau, le shingeki 新劇. Il décéda en 1919 de la grippe espagnole en pleine préparation de Carmen. Matsui Sumako qui tenait le rôle, se suicida le soir de la première, deux mois après la mort de son amant.

3. Cette exclusion décidée pour éviter qu'une prostitution non contrôlée se développe a entraîné celle des wakashū 若衆, les jeunes éphèbes qui furent à leur tour exclus de la scène. Jusqu'en 1891, les rôles féminins étaient tenus exclusivement pas des acteurs 
part, la naissance du théâtre moderne où, pour la première fois, entrait en scène une actrice professionnelle formée par une école d'art dramatique et, d'autre part, l'explosion des débats et des polémiques autour de la figure de la femme nouvelle.

D’abord présentée par la Société des arts littéraires (Bungei kyōkai) dirigée par Tsubouchi Shōyō (1859-1935) à l'université Waseda, la production ne sortait pas du cadre universitaire, mais la notoriété d'Ibsen, le jeu de l'actrice, très apprécié du public, et le message fort de la pièce créèrent l'événement, suscitant un grand écho dans la presse 4 . Un critique de théâtre, Ihara Seiseien 伊原青々園 (1870-1941) décrivant la réaction du public resté plutôt impassible jusqu'à la fin de la pièce, remarquait deux femmes, Okada Yachiyo 岡田八千代 (1883-1962) ${ }^{5}$ et Hasegawa Shigure 長谷川時雨 (1879$1941)^{6}$ qui sortirent leur mouchoir pour essuyer leurs larmes, lorsqu’à son mari Torvald Helmer qui s'exclamait : "J'aurais tout supporté pour toi, soucis et privations, mais il n'y a personne qui offre son honneur pour l'être qu'il aime », Nora répondit : "Des centaines de milliers de femmes l'ont fait $^{7}$ ". Ihara déclarait alors avoir découvert les vraies «femmes nouvelles " du Japon, celles qui s'inspiraient de personnages du théâtre moderne pour repenser leur vie de femmes (Rodd 1991).

masculins adultes, onnagata ou oyama 女形. À partir de 1891, se constitua une troupe, Misakiza 三崎座, composée exclusivement de femmes qui jusque-là avaient exercé comme professeurs de danse ou de chant de kabuki ou de kyōgen 狂言, mais la troupe disparut en 1915 (http://www.kanko-chiyoda.jp/tabid/332/Default.aspx [consulté en mars 2012]).

4. De nombreuses revues lui consacrèrent des numéros spéciaux : Shi to geki 詩と劇 (Poésie et théâtre), septembre 1911 ; Kabuki 歌舞伎, novembre 1911 ; Seitōo, janvier 1912. Il faut noter que Tsubouchi ayant vendu ses résidences pour investir dans la création de la salle de théâtre, était présenté comme un homme qui avait fait des sacrifices sur ses deniers personnels pour l'avenir du théâtre moderne au Japon.

5. Membre bienfaitrice de l'association Seitōsha, elle est aussi la sœur du dramaturge moderne Osanai Kaoru 小山内董 (1881-1928). Celui-ci l'avait enjointe lors de son mariage de renoncer à l'écriture au prétexte qu'au Japon une épouse ne pouvait pas être en même temps romancière.

6. Membre bienfaitrice de Seitōsha. Elle est déjà reconnue comme la première auteure dramaturge. Elle fonda plus tard la revue Nyonin geijutsu 女人芸術 (Femmes artistes) (1928-1932). Sa mère lui avait interdit de lire des livres.

7. Des centaines de milliers est la traduction française de Régis Boyer (1988) : certains commentateurs japonais considèrent la traduction en japonais " exagérée ". Au contraire, le terme de " milliers » reviendrait à minimiser l'intention de l'auteur. 




Fig. 1. Photomontage des scènes de Maison de poupée, avec Matsui Sumako dans le rôle de Nora, publié dans le numéro de la revue Taiyō de juin 1913 consacré aux femmes nouvelles. 
Mais que représentait le vocable de «femme nouvelle» ? On ne sait si cette expression alors à la mode désigne un fantasme ou une réalité. En 1910, le dramaturge Tsubouchi Shōyō, dans sa tournée de conférences intitulées " La femme nouvelle dans le théâtre moderne ${ }^{8}$ ", prononcées à Tokyo et dans les villes d'Osaka, de Kyoto, et de Kobe, souligne le caractère fantasmatique des descriptions dans les articles de presse où la femme nouvelle est tantôt idéalisée, tantôt vilipendée, tantôt critiquée pour son manque de féminité, ou au contraire accusée de développer à l'extrême des défauts typiquement féminins comme la préciosité ou le caractère capricieux. La figure de la dangereuse révolutionnaire n'est pas absente". Ainsi, il se demande si la "femme nouvelle " n'existerait pas que dans l'imagination de ceux qui défendent un idéal féminin, qu'il soit nouveau ou éternel. Cependant, de son côté, il se propose d'en dégager des traits saillants à partir des héroïnes des pièces de théâtre, Nora, Hedda Gabler, Magda, etc. Puis il s'interroge sur l'apparition ou non de ces types de femmes dans la société japonaise. Dans la presse, les articles sur les femmes nouvelles avaient commencé à fleurir, avant même la création de la revue Seitō 青鞜 (voir infra). Entre mai et juillet 1911, 35 articles, signés Kōtensei 晧天生 (nom de plume de Suzuki Bunji 鈴木文治 [1885-1946]), étaient dédiés à la femme nouvelle (atarashiki onna 新しき女) dans le quotidien Tōkyō Asahi shinbun 東京朝日新聞. Le premier article de la série présentait le mouvement féministe comme un mouvement inexorable à l'échelle mondiale. Le second poursuit en présentant les thèses et l'historique du féminisme à travers le monde. Le ton, bienveillant au départ, de ces articles se fait de plus en plus critique au fur et à mesure qu'ils concernent la réalité japonaise, en particulier lorsqu'ils abordent la question de la surveillance des fréquentations des lycéennes par les directeurs des établissements. Dans un des articles (du 24 mai, sous le titre "Nihon fujin no jikaku "日本婦人の 自覚 [La prise de conscience des Japonaises]), Hiratsuka Raichō 平塚らいてう (1886-1971) est citée comme un exemple connu, moralement condamnable, de ces femmes nouvelles. La Nora comme modèle de ces femmes nouvelles n'était pas inconnue au Japon.

8. Conférences qui seront publiées en avril 1912 (Tsubouchi 1912).

9. Kanno Suga 管野スガ, exécutée lors de la répression du crime de lèse-majesté (Taigyaku jiken 大逆事件) le 25 janvier 1911, est citée dans l'article de l'Asahi shinbun du 24 mai 1911, par une périphrase : « il y a même une femme dans cette effroyable affaire ». 


\section{Le prestige d'lbsen au Japon}

Le nom d'Ibsen avait été présenté pour la première fois au Japon par Mori Ōgai 森鴎外 (1862-1922) en 1889, puis par Tsubouchi Shōyō en $1892^{10}$. Une partie de la pièce Une Maison de poupée avait été traduite par Takayasu Gekkō 高安月郊 (1869-1944) en 1893. Celui-ci proposa au lectorat japonais une traduction entière des principales pièces sociales d'Ibsen, dès 1901. Une autre traduction d'Une Maison de poupée, de Fujisawa Kosetsu 藤沢古雪 ( ?- ?) était également éditée la même année sous le titre Ningyō no sumai 人形のすまみ ${ }^{11}$. Ibsen exerça une influence essentielle sur bien des auteurs, et non des moindres de cette époque. Après la mort d'Ibsen en 1906, ses œuvres furent systématiquement traduites et présentées, Yanagita Kunio 柳田国男 (1875-1962) et Iwano Hōmei 岩野泡鳴 (1873-1920) créèrent même une Association Ibsen (Ipusenkai イプセン会). D’entre toutes, Une Maison de poupée resta la pièce la plus traduite et retraduite, la plus représentée $^{12}$. Dès le 6 septembre 1906, les membres de la Société des arts littéraires de Waseda annoncèrent dans le journal Yomiuri shinbun 読売新聞 leur ambition d'en donner une représentation (Tsubouchi kyōkai 1998 : 130). Un premier débat autour de la question de la fugue de Nora avait été lancé en $1906^{13}$, lors du boom Ibsen, avec la publication d'un article de Kuwaki Gen.yoku 桑木厳翼 (1874-1946), professeur de philosophie à l'Université impériale de Tokyo (Kuwaki 1906) ${ }^{14}$.

10. Dans Waseda bungaku 早稲田文学, $\mathrm{n}^{\circ} 28$, sous le titre «Ibusen no kigeki » イブセンの 喜劇 (Les drames d'Ibsen), il donne un résumé de la pièce Une Maison de poupée.

11. Cité sur le site http://homepage3.nifty.com/nada/ibsen.html (consulté le 26 juin 2012).

12. On trouvera la liste des traductions et des éditions : http://homepage3.nifty.com/ nada/ibsen.html (consulté en juillet 2012).

13. Hiratsuka Raichō lut à cette époque l'article (Hiratsuka 1992, vol.1 : 178 et vol.2 : 12). Elle indique également avoir lu la pièce traduite en japonais (dont elle a oublié le nom du traducteur) à la suite de cette lecture.

14. C'est à cette occasion que Lu Xun 鲁迅 (1881-1936), qui avait séjourné au Japon entre 1902 et 1909, découvrit le théâtre d'Ibsen, en particulier Un Ennemi du peuple (1881). À ce stade, il ne s'intéressa guère au personnage de Nora et c'est seulement après le Mouvement du 4 mai 1919 que Nora devint synonyme de la femme nouvelle en Chine. C'est le philosophe Hu Shi 胡適 (1891-1962) devenu un des intellectuels le plus en vue, qui, influencé par la réception d'Ibsen aux États-Unis dont il revenait, 
Ce fut donc un événement auquel le public était préparé depuis plusieurs années, et quelques mois avant novembre 1911, lorsque la presse publiait des articles sur Nora et la pièce, elle annonçait que celle-ci serait jouée à guichets fermés (Hiratsuka 1992, vol. 1 : 131). Cette attente permet de mieux comprendre la rapidité avec laquelle la revue Seitō qui venait de voir le jour en septembre 1911 décida de consacrer un numéro spécial à cette pièce.

\section{Échos d'Une Maison de poupée}

Une Maison de poupée est en réalité la seconde représentation au Japon du théâtre d'Ibsen, la première ayant eu lieu en 1909 avec la mise en scène par Osanai Kaoru de la pièce John Gabriel Borkmann (1869) traduite par Mori Ōgai. Si elle fit date dans l'histoire du théâtre au Japon, elle fut loin d'avoir rencontré l'écho d'Une Maison de poupée qui devait beaucoup à l'art et au succès de Matsui Sumako certes, mais qui montrait surtout l'intérêt naissant pour la question féminine. L'illustration concrète de cette nouvelle tendance, c'était la création de l'association Seitōsha 青靱社, créée uniquement par des femmes deux mois plus tôt, en juin 1911.

Hiratsuka Raichō (infra Raichō), initiatrice et membre fondatrice de cette association, lança un appel à communications à ses consœurs dans le numéro de novembre 1911, dans le but non pas d'échanger de simples impressions ou des critiques artistiques, mais pour étudier véritablement les conséquences et les significations de cette pièce. Elle invitait toutes les membres de Seitō à proposer des contributions personnelles, en précisant ce qui devait être l'objet de réflexions :

défend le personnage de Nora dans la revue Xinn Qingnián 新青年 La Jeunesse (littéralement : Nouvelle Jeunesse). Celle-ci consacra un numéro spécial en juin 1918 à Ibsen. Le personnage de Nora devint le symbole des étudiantes du 4 mai. C'est en réponse à Hu Shi que Lu Xun écrivit une suite à Une Maison de poupée, dans laquelle il exprimait son pessimisme quant au destin de la Chine, symbolisée par l'impasse dans laquelle se retrouverait Nora après avoir abandonné le foyer (Lu 1981). En Chine, la pièce garda un prestige immense par la suite. Voir le " "Problème des femmes" dans La Nouvelle Jeunesse 1915-1922 " de Cini Francesca (http://www.afec-en-ligne.org/IMG/pdf/5Cini.pdf [consulté en juillet 2012]). Voir également l'article de Niimura Yōko dans ce même dossier. 
1. La Nora inconsciente

2. Le processus de sa prise de conscience

3. La Nora consciente

4. Le futur de Nora (la Nora qui apparaît dans Une Maison de poupée au moment de sa déclaration d'indépendance est en fait un simple point de départ, et soulève beaucoup de questions aujourd'hui à nous les femmes)

5. L'opinion sur l'ensemble

Elle ajoutait à ces " consignes » une bibliographie de vingt-six titres d'ouvrages étrangers, à commencer par les pièces de George Bernard Shaw (1856-1950), ainsi que douze titres japonais, parmi lesquels la traduction d'Une Maison de poupée de Shimamura Hōgetsu de 1910, des pièces sociales d'Ibsen par Takayasu Gekkō, et des articles sur Nora, sur la femme nouvelle. Parmi ces derniers, elle cite l'article de Kuwaki Gen.yoku (voir supra), dans lequel celui-ci critique l'éducation tournée vers le principe de la «bonne épouse, mère avisée " (ryōsai kenbo 良妻賢母) $)^{15}$, qui a pour conséquence, selon lui, de nier la personnalité de la jeune fille et de provoquer inévitablement des conflits semblables à ceux entre Helmer et Nora.

Il ne faut pas oublier que ces critiques sur le caractère contraignant et négatif de l'enfermement dans un rôle familial étaient bien minoritaires dans la société. Car après que le gouvernement eut adopté un décret interdisant aux femmes la participation aux activités politiques en 1890, il prescrit le principe de la formation de la " bonne épouse, mère avisée ". Avec le décret promulgué en 1899, pour faire face à l'influence des écoles des missionnaires, cette idéologie devint officielle, et le ministre de l'Éducation, Kabayama Sukenori 樺山資紀 (1837-1922), artisan de ce décret, déclarait que le rôle de l'enseignement secondaire pour les filles était de former des bonnes épouses et des mères avisées pour le progrès de la société.

15. Mori Arinori 森有礼 (1847-1889) aurait été le premier ministre de l'Éducation (1885) à l'utiliser d'après Haga Noboru 芳賀登 (1990 : 8). Le concept de ryōsai kenbo était nouveau par rapport à la tradition confucéenne, dans la mesure où il a été élaboré dans l'idée de donner la responsabilité à la mère d'éduquer la génération future dans la perspective de la construction d'un État et d'une nation modernes. Le premier à l'avoir introduit est Nakamura Masanao 中村正直 (1832-1891), dans la revue Meiroku zasshi 明六雑誌 (Revue de l'an 6 de Meiji), comme symbole de civilisation. Dans les familles de guerriers avant Meiji, les garçons étaient séparés des pièces réservées aux femmes dans la maison, donc de leur mère, à l'âge de sept ans. 
Le taux de scolarisation des filles dans l'éducation élémentaire, qui atteignait à peine $30 \%$ en 1890, avait progressé régulièrement depuis 1897 , passant de $50 \%$ à $96 \%$ en 1907 . Mais la diffusion de l'éducation à travers le pays s'était accompagnée d'une fixation des rôles sexués au sein de la famille et de la société, par exemple des cours de couture furent introduits plus systématiquement dans les écoles pour convaincre les familles de l'utilité d'y envoyer leurs filles (Joseishi sōgō kenkyūkai 1982, vol. 4 : 152). Cette stratification genrée dans les écoles vit son décret d'application en 1900 dans un règlement du ministère de l'Éducation. L'article premier stipulait qu'une éducation appropriée à chaque sexe devait être dispensée en tenant compte de leurs rôles futurs respectifs. Ce qui impliquait une morale plus stricte pour les filles, le choix de textes de lecture où il était question des tâches ménagères pour les filles, la non mixité des classes, et l'introduction de matières utiles aux filles, comme la couture. Ces mesures furent appliquées avec beaucoup d'attention et les discours successifs des ministres de l'Éducation allèrent dans ce sens.



Fig. 2. Couverture de la revue Seitō, mai 1912. Bibliothèque de l'université Waseda. 
La difficulté paraissait plus complexe encore pour les autorités à ses divers échelons - patriarcal, étatique ou sociétal - lorsqu'il s'agissait de maintenir les jeunes filles à leur place lorsqu'elles recevaient une éducation supérieure. L’idéologie de la " bonne épouse, mère avisée " devait être un instrument pour empêcher que les filles qui suivaient une éducation supérieure ne deviennent insolentes, comme le déclarait explicitement le ministre de l'Éducation en 1906 :

« [...] il y a des femmes qui aspirent à embrasser une carrière et à trouver un emploi après leurs études, mais cela doit rester une exception, car leur vocation demeure avant tout dans le fait de devenir l'épouse d'un homme, de gérer le foyer et d'éduquer les enfants. » (Fukatani 1998 : 156).

Dans un tel contexte, on s'en doutera, nombreuses étaient les professeures de lycées pour filles qui mettaient en garde leurs élèves contre la lecture et le spectacle de cette pièce d'Ibsen, comme en témoigne Otake Kōkichi (Beniyoshi) 尾竹紅吉 (1893-1966) dans le numéro de Seitō publié en mai 1912, dans lequel elle donne non seulement l'appréciation de la représentation qu'elle a vue à Osaka - jouée par la même troupe - mais rapporte aussi les propos recueillis auprès d'autres spectatrices ${ }^{16}$.

La représentation de la pièce suscita de nombreuses réactions à travers la presse féminine de l'époque et dans le monde académique. Des discussions s'en étaient suivies dans diverses revues, par exemple dans la revue Fujin kurabu 婦人俱楽部 (Le Club des femmes) qui publia un article de Sawada Bushō 沢田掹松 (1871-1927), et les journaux relataient les conférences du professeur Ukita Kazutami 浮田和民 (1860-1946), tous deux prêts à reconnaître l'importance de l'éveil (kakusei 覚醒) de la femme, mais interprétant la pièce comme un avertissement donné aux jeunes filles pour qu'elles ne tombent pas dans un individualisme menaçant le maintien des liens familiaux (Lowy 2007 : 28-31).

16. " $\mathrm{C}$ et $\mathrm{D}$ sont encore des lycéennes et elles avaient reçu un avertissement de leur proviseur de ne pas assister à Une Maison de poupée " écrit-elle (Seitō mai 1912). Voir également Shin Nihon bungaku-shi 新日本文学史 (Nouvelle histoire littéraire du Japon), Yorozu chōhō 萬朝報, 1926. Le contraste entre Tokyo et Osaka dans la réception de la pièce qui s'en dégage est frappant. Il est corroboré par les propos qu'on peut trouver dans la presse de la ville d'Osaka (Tsubouchi kyōkai 1998 : 180-184). 
La pièce avait déjà été traduite et était donc connue au moins partiellement par une élite intellectuelle. Raichō, comme nous l'avons déjà signalé, avait été dès 1906 sensibilisée à la cause féministe précisément par la lecture d'un article présentant cette pièce. D'ailleurs, dès le premier numéro, en septembre, elle publia dans la revue Seitō un article sur Hedda Gabler (1890). Et avant même que cette revue n'y consacre son supplément spécial de janvier 1912, la revue Joshi bundan 女子文壇 (Le Monde littéraire des femmes), en janvier et en octobre 1909, avait soulevé la question de l'autonomie économique de la femme et citait, dans un des articles, l'exemple à ne pas suivre, celui de Nora. En novembre 1909, un article intitulé "Les difficultés du célibat féminin " critiquait la mode et l'aspiration parmi les jeunes filles cultivées à vouloir vivre comme des Nora d'Ibsen (Tanaka 2005). La représentation de la pièce et le débat autour de Nora eurent sur cette revue un double effet : d'une part l'augmentation de la proportion de contributions écrites par des femmes à partir de janvier 1913, et d'autre part la publication d'articles sur la question de l'indépendance économique de la femme et sur la femme nouvelle. Cet intérêt croissant pour la pièce d'Ibsen et la femme nouvelle amena la revue Chūō kōron à publier un dossier spécial consacré à Hiratsuka Raichō (sous le titre " Hiratsuka Haruko-ron » 平塚 明子論), en juillet 1913, comme la représentante des " Nora japonaises » (wasei Nora 和製ノラ ${ }^{17}$ ). Ceci nous permet d'affirmer que malgré les attaques dont elle fut l'objet, la revue bénéficiait d'une certaine reconnaissance pour sa valeur avant-gardiste. Par ailleurs de nombreuses lectrices ou auteures d'articles de Joshi bundan soutinrent activement Seitō ${ }^{18}$. À l'heure où certains titres attaquaient de front la Nora d'Ibsen ${ }^{19}$, cette revue avait évolué plus favorablement envers Nora et alla jusqu'à publier des articles après

17. Littéralement de "fabrication japonaise ». La presse qualifiait l'association de " pépinière des Nora japonaises " (wasei Nora yōseijo Seitōsha 和製ノラ養成所青鞜社), pour désigner l'association Seitōsha (Hiratsuka 1992, vol. 2 : 34).

18. Parmi les plus connues d'entre elles, citons Harada Kotoko 原田琴子 (1892-1973), Ikuta Hanayo 生田花世 (1888-1970), Katō Midori 加藤みどり (1887-1919), Mikajima Yoshiko 三力島臀子 (1886-1927), Mizuno Senko 水野仙子 (1888-1919), Mochizuki Rei 望月麗 (1893-1943), Yamada Kuniko 山田邦子 (1890-1948).

19. Notamment Fujin mondai 婦人問題 (La question féminine) d'Uesugi Shinkichi 上杉慎吉 (1878-1929), en novembre 1910, Joshi kyōiku 女子教育 (L'éducation des filles) de Yoshida Kumaji 吉田熊次 (1874-1964), décembre 1911. 
1911 dans lesquels Nora était considérée comme le symbole de l'aspiration à la liberté.

Avant de présenter et de commenter le numéro spécial consacré à Une Maison de poupée par la revue Seitō, rappelons l'enjeu féministe de cette pièce maîtresse d'Ibsen.

\section{Le « crime » de Nora}

Maîtresse de maison, dévouée, gaie, soumise, Nora, l'héroïne de la pièce, apparaît comme une femme-enfant, insouciante, dont le bonheur consiste à aimer et à être aimée, à jouir de la présence de ses enfants et de son mari. Son mari, Helmer, l'apprécie ainsi et lui donne des surnoms comme " petit écureuil », "petite alouette».

Au début de la pièce, Nora confie à son amie d'enfance, Kristine Linde, son grand secret : elle avait contrefait la signature de son père, âgé et infirme, dans le but d'emprunter de l'argent à un usurier et de permettre ainsi à son mari, malade lui aussi à ce moment-là, de partir en voyage de cure. D'autre part, elle a menti à son mari sur l'origine de cet argent car Helmer, tout en ignorant la gravité de son état, n'aurait point approuvé l'emprunt : elle lui dit alors qu'elle avait reçu cette somme en cadeau de son père.

Nora était très fière de son acte. Dans son esprit elle avait réussi à la fois à épargner son père de la voir aux prises avec de tels problèmes et à sauver la vie de son mari. Il y avait un autre motif d'orgueil pour Nora : en effet, pour payer l'usurier elle devait épargner sur ses dépenses personnelles et, en plus, elle devait travailler en secret pour se procurer un peu plus d'argent. Ibsen avait écrit dans sa première esquisse de la pièce, en octobre 1878 :

"Elle a commis un faux, et c'est sa fierté ; car elle l'a fait par amour pour son mari, afin de sauver sa vie. Mais cet homme, avec toute sa loyauté banale, se tient sur le terrain de la loi, et considère la question d'un œeil masculin » (Braunschweig 2009/2010 : 10).

Dans cette phrase se trouve exprimé clairement le projet de la pièce, à la fois plaidoyer de la cause féministe, mais aussi critique de la médiocrité et de l'enfermement de l'individu dans les conventions. Chez Ibsen, le féminisme constitue un aspect particulier de l'individualisme. Les dernières répliques de la pièce font apparaître avec clarté sa pensée lorsqu'il fait dire à Nora : "J'ai d'autres devoirs qui ne sont pas moins sacrés... les devoirs 
envers moi-même ». Dans la fin de la pièce, il ne s'agit plus seulement de la situation juridique - mineure - de la femme, mais de sa condition de femme en général et de son passage à sa réalisation en tant qu'individualité humaine où ne devrait plus jouer la double morale, celle du droit naturel auquel la femme a souvent tendance à tendre dans sa spontanéité, et celle de la législation en vigueur dans les pays occidentaux, en particulier le Code Napoléon qui a tant influencé les législations familiales européennes. La double morale ne provient pas d'une différence de nature, mais de la différence de la situation faite à la femme, de l'infériorité sociale et juridique vis-à-vis de l'homme.

\section{Une Maison de poupée et le féminisme en Norvège}

Lorsque Ibsen publie sa pièce en 1879 , le féminisme n'est plus seulement alors une foi un peu vague qu'on garde dans son cour, mais un mouvement qui présente un programme, des revendications. Ibsen se place dans la lignée du féminisme norvégien de son époque et plaide en faveur d'une réforme du régime matrimonial. Il a payé de sa personne avant et après, par exemple en obtenant, en 1879, au club scandinave de Rome la modification des statuts pour que les femmes aient le droit de vote dans ses assemblées et que la bibliothécaire puisse être une femme. Avec cette pièce, il ouvre en Norvège une campagne pour généraliser le régime de la séparation des biens, se conformant aux indications que donne Stuart Mill (18061873) dans son manifeste sur la servitude des femmes (1851). Cinq ans plus tard, en 1884, Ibsen s'associe aux trois autres grands auteurs radicaux, Bjørson (1832-1910), Lie (1833-1908) et Kielland (1849-1906), pour présenter une supplique à la commission de Justice au Parlement ${ }^{20}$ pour que le régime de séparation des biens devienne systématique et que la femme soit reconnue majeure dans l'état de mariage.

20. La Norvège, enlevée au Danemark au Congrès de Vienne en 1814, fut attribuée à la Suède, mais les Norvégiens refusèrent le pacte et proclamèrent leur indépendance. Par l'acte d'union de 1815, elle obtenait une semi-indépendance, et elle posséda sa propre armée, sa marine, ses services de douanes, son parlement et une certaine autonomie à l'intérieur de ses frontières. La rupture définitive de l'union avec la Suède intervint en 1905. 
À une époque où une femme ne pouvait guère prendre la plume sans choisir un pseudonyme masculin ou l'anonymat, les féministes en Norvège avaient commencé à publier des pamphlets et des romans qui ont grandement influencé Ibsen. En 1871, Mathilde Schjøtt (1844-1926) publie, sans nom d'auteur, Venindernes samtale om Kvindens Underkuelse (Conversation de trois amies sur la sujétion de la femme). Camilla Collett (1813-1895), militante féministe et auteure des Filles du préfet (Amtmandens døttre,1854), s'était liée d'amitié avec Henrik Ibsen et avait rendu visite au couple à Munich en 1876. Ses idées inspirèrent grandement le dramaturge. Ibsen avait jusque-là, chez ses héroïnes des pièces historiques, exalté des caractères que l'on ne considérait guère comme féminins, comme la volonté tendue à l'extrême, la volonté d'indépendance exaltées dans les sagas islandaises et norvégiennes à une époque où le christianisme n'avait pas encore imposé la servitude de la femme. Mais, il adopte à partir de 1875 des thèmes chers à Camilla Collett et avec les Soutiens de la société qui paraît en 1877, il se lance dans une confrontation ouverte aux problèmes contemporains (Gravier $1968: 79$ sq.).

Le thème fondamental d'Une Maison de poupée est le désenchantement de la femme après le mariage, sa cruelle désillusion quand elle découvre le véritable visage de son mari. Comme l'écrit Claudio Maigris (Braunschweig 2003), le rêve d'une humanité heureuse et réconciliée avec elle-même que la civilisation rend impossible, il le poursuit de pièce en pièce, jusque dans la désillusion. L'anecdote d'Une Maison de poupée n'est pas inventée de toutes pièces. Ibsen a recueilli les confidences de Laura Kieler ${ }^{21}$, épouse malheureuse, victime de son dévouement et de sa naïveté. Néanmoins, il a

21. Admiratrice d'Ibsen, elle avait écrit une suite à Brand. Bien qu'ayant eu une enfance malheureuse comme un des personnages de la pièce, Kristine Linde, elle était insouciante et on l'appelait " l'alouette ». Comme Nora dans la pièce, elle contracta un emprunt pour permettre à son mari malade des poumons de se faire soigner en Suisse et en Italie. Elle rend visite aux Ibsen pour leur parler des angoisses que lui cause cette dette. Ibsen lui conseille de tout dire à son mari, mais elle ne le suit pas et falsifie une traite qu'elle n'avait pu payer. Un riche parent la dénonce et le scandale éclate. Son mari la fait interner dans un hôpital psychiatrique et demande le divorce. L'histoire de Laura fournit donc le point de départ de l'intrigue. Camilla Collett lui reprocha d'avoir utilisé des personnages qu'il n'était pas difficile d'identifier dans un pays faiblement peuplé comme la Norvège. 
stylisé l'histoire, l'a rendue moins déprimante, car Laura avait affaire à un mari brutal et psychopathe. Et au lieu de l'achever comme une pièce victorienne $^{22}$ où après une dispute les époux auraient retrouvé leur confortable existence bourgeoise, le grand revirement se produit lorsque Nora reçoit son pardon, mais qu'elle rejette avec tristesse l'indulgence de son mari et que sonne pour Helmer stupéfait l'heure de vérité.

Comment la femme-enfant qui grignote des macarons en cachette, s'amuse sans contrainte avec ses enfants et dresse joyeusement le sapin de Noël, en vient-elle à déchirer si vite et si complètement le voile de ses illusions ?

Bien des objections ont été formulées à l'égard de la dernière scène, et nous en retrouvons quelques unes dans le débat qui a animé les membres de la revue Seitō, en particulier de la part de Hiratsuka Raichō qui lui préféra des pièces comme Hedda Gabler ${ }^{23}$.

Mais ces objections ne se présentent à l'esprit du spectateur que lorsqu'il a quitté le théâtre, après une méditation sur la pièce. Au théâtre, la magie opère et Ibsen nous impose sans peine sa fiction hardie.

Avec son intrigue conventionnelle et l'intervention du maître chanteur Krogstad, Une Maison de poupée risquait d'être classée dans le répertoire des mélodrames. En mettant son œuvre au service d'une cause généreuse, Ibsen aurait pu la hausser simplement au niveau de la pièce à thèse. Mais au moment où Nora, refusant d'entretenir plus longtemps ses propres illusions, crie sa déception et son dégoût à Helmer, le frisson tragique parcourt la salle et saisit d'effroi le spectateur. Nora pousse la sincérité jusqu'à l'héroösme. Son départ est un saut dans l'inconnu. Elle affirme son moi en détruisant son bonheur, et devient à ce titre une héroïne tragique. Ce n'est pas seulement parce qu'elle brave les conventions et les autorités. Ibsen nous arrache aux préoccupations sociales et devient le poète qui nous éloigne même du sujet de la guerre des sexes, pour nous placer face à une question existentielle de la connaissance et de la communication avec autrui.

Si cette pièce a connu un si grand succès sur le plan mondial, c'est probablement parce qu'Ibsen expose la revendication en termes généraux de réalisation d'individualité humaine qui résonne dans le cœur de chacun.

22. Ce qui sera imposé dans les premières versions allemandes, voir infra.

23. L'effet de choc du changement soudain dut être d'autant plus grand que le second acte fut non pas joué, mais raconté et expliqué par Shimamura Hōgetsu. 


\section{La réception de la pièce à travers le monde}

En Europe comme aux États-Unis, au XIx ${ }^{\mathrm{e}}$ siècle, la pièce fit scandale. Elle fut d'abord interdite en Grande-Bretagne par le Lord Chamberlain sous couvert de l'acte de censure de $1737^{24}$. Pour sa première représentation en Allemagne, en 1880, Ibsen dut céder à l'exigence de l'actrice principale et modifier la fin : Helmer la traîne dans la chambre des enfants où en les voyant, elle s'effondre, et le rideau est abaissé. Ibsen regretta amèrement d'avoir cédé, et qualifia cette fin de honteuse, d' " attentat barbare » au texte original. Jusqu’à la fin des années 1890 , le public allemand ne connut que cette fin qui avait transformé la pièce en un drame sentimental bourgeois d'intrigue et de réconciliation ${ }^{25}$. Dans les capitales norvégienne et suédoise, les cartons d'invitation de la bonne société étaient suivis d'une précision : "On est prié de ne point discuter d'Une Maison de poupée ".

Pourtant, Ibsen qui avait écrit dans la première esquisse de cette pièce qu' « une femme ne peut pas être elle-même dans la société contemporaine, c'est une société d'hommes avec des lois écrites par les hommes, dont les conseillers et les juges évaluent le comportement féminin à partir d'un point de vue masculin " (Braunschweig 2009: 6) ne songea plus à défendre la cause des femmes après que celle-ci aura remporté ses premières victoires en Norvège. Il peindra d'autres types de femmes et peut-être pour cette raison, on a retenu de lui ses propos lors de son toast aux féministes norvégiennes, rapportés d'un livre à l'autre :

"Je ne fais partie d'aucun comité féministe. [...] Je suis plus poète que philosophe et je décline l'honneur d'avoir lutté consciemment pour la cause féministe. [...] Pour moi, il n'y a qu'une cause, la cause de l'humanité $[\ldots]^{26}$."

Il faut y voir la méfiance d'un homme vis-à-vis de la société et de toutes ses institutions, d'un homme croyant avant tout à l'individu, qui ne veut se laisser aveugler par aucun parti pris, ni être dupe d'aucun discours préconçu.

24. La première représentation date de 1889.

25. Le féminisme en Allemagne ne présenta pas avant 1902 la revendication du suffrage pour les femmes, alors que cette revendication avait débuté en Angleterre en 1867.

26. Publié dans La Fronde, le 16 juin 1898. Mise en ligne le 2 septembre 2002 : http:// www.youscribe.com/catalogue/presse-et-revues/actualite-et-debat-de-societe/medias/ la-fronde-numero-190-du-16-juin-1898-893827 
Au Japon, Shimamura Hōgetsu qui revenait d'un séjour d'études en Allemagne, avait proposé sa première traduction de la pièce en 1906 à partir d'une édition allemande populaire, mais il reprit le texte original à partir de la version anglaise, en 1910, préparant le public à la représentation avec la fin que nous connaissons ${ }^{27}$.

Cette pièce bouleversa le théâtre et devint le symbole du théâtre occidental moderne posant la question de la liberté existentielle. Elle fut utilisée comme l'emblème de la lutte des femmes pour leur liberté et l'égalité. Il n'est donc pas surprenant qu'elle servit à introduire à la fois la première pièce du théâtre moderne et le premier débat public sur la question de la femme nouvelle, sujet de controverses jusque chez les membres de la revue Seitō qui venait tout juste de voir le jour. Rappelons brièvement le contexte de l'époque pour situer la revue.

\section{Le contexte idéologique et littéraire au moment de la parution de Seitō}

L'année 1911 s'ouvrit par l'exécution de douze des vingt-quatre militants socialistes et anarchistes condamnés à mort lors de l'Affaire du crime de lèse-majesté (Taigyaku jiken 大逆事件) ${ }^{28}$. La censure politique fut systématiquement appliquée à toute œuvre ou article qui évoquait le socialisme ou l'anarchisme. Le 3 septembre 1910, cinquante-six ouvrages furent interdits pour " pensée destructrice" (hakai shiso 破戒思想). La nouvelle traduc-

27. Kano Ayako (2001 : 186-187) parle de cette fin allemande, mais ne mentionne pas les deux traductions de 1901 qui présentaient déjà la fin originale d'Ibsen au Japon. La traduction de Hōgetsu est éditée par la maison d'édition de l'université Waseda en 1913, avec une introduction.

28. La plus connue des quatre affaires de crime de lèse-majesté est désignée aussi sous le nom de Kōtoku jiken 幸徳事件. Le procès fut mené à huis clos, sans témoin et dans un grand empressement. Il s'ouvrit le 20 décembre 1910 pour aboutir, le 18 janvier 1911, à la sentence capitale de vingt-quatre accusés et à la condamnation des deux autres militants à onze ans et huit ans de prison ferme pour détention d'explosifs. Le lendemain, douze des condamnés virent leur peine commuée en travaux forcés à perpétuité par une grâce impériale, comme si tout était prévu dès le départ. Les autres furent tous exécutés dans la semaine qui suivit, dans une précipitation sans précédent dans l'histoire judiciaire du Japon moderne (Lévy 2010). 
tion ${ }^{29}$ par Kawada Shirō 河田嗣郎 (1883-1946) de l'ouvrage De l'assujettissement des femmes (1869) de J. S. Mill, pourtant déjà traduit ${ }^{30}$ en 1878, fut interdite.

Le ministre de l'Intérieur, Hirata Tōsuke 平田東助 (1849-1925), dans un rapport adressé au chef du gouvernement en juillet 1910, considérait que l'influence exercée par les ouvrages politiques sur la jeunesse était un facteur bien plus dangereux que les différenciations en classes sociales provoquées par la modernisation, et préconisait des mesures de prévention dans l'enseignement. Malgré l'atmosphère répressive et le poids du nationalisme de cette époque, de nouvelles œuvres littéraires furent traduites et introduites en grand nombre, du russe, du français, du norvégien ou du suédois. Le roman naturaliste, qui avait été un des principaux courants littéraires, fut relayé, en 1910, par la revue Shirakaba 白樺 (Bouleau blanc) marquée par l'idéalisme, l'humanisme et l'individualisme.

Pour prémunir la jeunesse et les femmes contre cette influence, les milieux scolaires et féminins furent l'objet de la plus grande attention du gouvernement qui élabora des directives successives en insistant sur l'enseignement moral, l'inculcation des principes traditionnels de piété filiale ${ }^{31}$ et le renouvellement des thèses de la formation de la «bonne épouse, mère avisée $»^{32}$. En 1911, le ministère de l'Éducation fait adopter à la Diète un mémorandum sur la promotion de l'enseignement moral auprès du peuple (Kokumin dōtoku kyōiku shinkō no kengi 国民道徳教育振興の建議), afin d'organiser, en particulier dans les écoles normales, des sessions de formation morale des futurs enseignants. Au même moment, la Diète adopta un texte sur l'éducation des filles, "Les dix préceptes moraux pour les filles " (Jokun jūkajō 女訓十ヶ条, cité par Hiratsuka Raichō, 1992, vol. 1 : 369).

Pour beaucoup de jeunes filles qui avaient eu la chance d'accéder à l'enseignement supérieur, cette éducation pétrie de morale avait représenté une grande déception, comme l'explique Hiratuska Raichō dans son

29. Sous le titre Fujin mondai 婦人問題 (La question féminine) chez Ryūbunkan 隆文館. 30. Sous le titre Danjo dōkenron 男女同権論 (Mill 1878).

31. Uesugi, Yoshida, voir note 19.

32. Shimoda Utako 下田歌子 (1854-1936) publie Fujin no jöshiki 婦人の常識 (Le bon sens des femmes) en 1910, ouvrage dans lequel elle prône un enseignement moins étroit pour élargir l'amour que porte la femme à la patrie et non plus seulement au foyer, mais elle y réaffirme que la vocation naturelle de la femme est de tenir son foyer. 
autobiographie. La méfiance à l'égard de l'éducation supérieure donnée aux filles de l'élite était grande en cette période de répression idéologique (Joseishi sōgō kenkyūkai 1982 :172) et la littérature devint la seule vocation autorisée pour celles qui bénéficiaient d'un enseignement universitaire. Pour cette raison, elle servit de point de départ pour exprimer leurs conflits intérieurs, leurs tourments et l'éveil de leur conscience. Leurs espoirs se tournèrent vers cette perspective, à la fois pour échapper à la dépendance et obtenir la reconnaissance de leur personnalité. Née dans ce contexte, la revue Seitō attira l'attention et la sympathie d'un très grand nombre de ces femmes. Elle leur permit de faire entendre leurs voix non sans susciter parfois des réactions violentes dans l'opinion publique, pas toujours prête à approuver l'idée de l'émancipation de la femme.

\section{Le numéro spécial consacré à Nora}

Le numéro de la revue Seitō de janvier 1912 comprend deux parties : une première consacrée aux nouvelles et poèmes, et une seconde constituée d'un supplément Nora (Furoku Nora 付録ノラ). Les deux tiers du mensuel (de la page 62 à la page 173) sont dédiés à la pièce d'Ibsen, et précisément à Nora. Dans la postface, en dernière page, Raichō se félicite du succès rencontré par le projet et explique que toutes les contributions, nombreuses, n'ont pu y être publiées, entre autres pour éviter les répétitions, précise-t-elle, tout en regrettant vivement de n'avoir pu y insérer l'article d'Okada Yachiyo 岡田八千代 (1883-1962) faute de l'avoir reçu à temps. Le supplément s'ouvre sur un extrait de l'ébauche notée par Ibsen en 1878 que nous avons déjà cité, sur la critique du jugement d'un point de vue masculin.

Suivent les cinq articles rédigés par des membres de l'association Seitōsha, Ueno Yō 上野葉 (1886-1928), Katō Midori, Ueda Kimi 上田 君 (1886-1971), toutes trois déjà mariées et mères de famille, Hiratsuka Raichō et Yasumochi Yoshi 保持研 $(1885-1947)^{33}$, ces dernières étant alors encore célibataires.

33. Elle signait ses articles du nom de plume de Hakuu 白雨 (Pluie blanche). 
À ces articles s'ajoutent deux traductions de critiques littéraires sur la pièce, celle de G. Bernard Shaw et celle de Jeannette Lee (1860-1951) ${ }^{34}$, un article anonyme ${ }^{35}$ dans lequel l'auteur présente une pièce française, $L a$ Révolte (1870) d'Auguste de Villiers de L'Isle-Adam, pour sa similitude avec le thème d'Une Maison de poupée ${ }^{36}$; un petit article de l'actrice Matsui Sumako qui explique les principales difficultés qu'elle a rencontrées pour jouer la pièce.

Le numéro est illustré par des photos : les portraits des actrices Matsui Sumako, Paula Somary et de l'actrice allemande Agnès Sorma (1862$1927)^{37}$ en Nora, ainsi que de deux photos de scène de théâtre, l'une au Japon, l'autre aux États-Unis.

En lançant ce numéro, la revue Seitō créa un événement, car si ni Nora ni la femme nouvelle n'étaient inconnues, en suscitant un débat entre les femmes elles-mêmes, la revue démontrait la volonté que celles-ci avaient désormais de s'approprier un "modèle " qu'elles allaient interpréter à leur façon et non selon les préceptes des éducateurs ou éducatrices reconnus dans leurs positions officielles. Un an plus tard, Seitō consacra deux numéros successifs, en janvier et février 1913, à la question des femmes nouvelles pour répondre aux attaques dont elles devinrent l'objet.

34. D'après les recherches récentes, notamment d'après l'ouvrage de Nakamura Toshiko (1997). Voir également : http://crd.ndl.go.jp/GENERAL/servlet/detail. reference? $\mathrm{id}=1000029320$

35. Hiratsuka Raichō ne réussit pas à se souvenir qui avait écrit l'article mais elle pense qu’il s'agissait de Sōma Gyofū 相馬御風 (1883-1950) ou de Kawatake Shigetoshi 河竹 繁俊 (1889-1967) (Hiratsuka 1992, vol. 2 : 13).

36. La différence est de taille, car l'héroïne qui plaque tout, son statut d'épouse et de mère parfaite, retourne résignée dans son foyer. C'est un vaudeville qui n'a tenu l'affiche que cinq jours à l'époque. Il a été rejoué en 2006, mais la critique fut médiocre.

37. Ce sont toutes les deux des actrices renommées qui ont joué le rôle de Nora. Un article décrit l'enthousiasme que suscite la réapparition d'Agnès Sorma dans Une Maison de poupée en 1898 : http://query.nytimes.com/gst/abstract.html?res=9B07E7D71638E 433A25756C1A9659C94699ED7CF (consulté le 7 juin 2012). 


\section{Les discussions entre les membres de Seitō}

\section{Sympathie et enthousiasme}

Les contributrices, à l'exception de Raichō, expriment leur enthousiasme et leur sympathie pour Nora. Ueno Yōko, enseignante depuis 1908, avait déjà rédigé un article en 1910 pour critiquer ouvertement l'inculcation du principe de «bonne épouse, mère avisée » dans les écoles. D’après les souvenirs d'Oku Mumeo 奥むめお (1895-1997) qui l'avait eue comme professeur de lycée, elle était connue pour y insuffler l'esprit de la " femme nouvelle " et son texte reflète son engagement et son enthousiasme.

Dans son texte comme dans les autres, l'acquisition d'une personnalité autonome par rapport à l'homme au sein du couple est considérée comme le message essentiel. Jiga 自我, jiko 自己, l'" ego», le «soi " sont les mots clés qui sont objet de discussions et de développements au sein de l'association. Katō Midori insiste aussi dans son texte essentiellement sur la question de l'acquisition d'une personnalité authentique. Admirant sa résolution de quitter le foyer, Katō Midori exprime, comme Ueno Yōko, sa sympathie pour Nora et identifie son geste à la révolte féminine : "Dans notre pays, au Japon, il y a bien des Nora qui n'ont pas encore pris conscience et parmi celles qui ont pris conscience, nombreuses sont celles qui partagent les tourments de Nora " (Horiba 1991 : 55).

Mais c'est surtout la discussion autour de l'image du couple moderne idéal, l'importance à accorder au mariage d'amour, qui caractérise ces textes.

\section{Les considérations sur le mariage}

Pour Ueno Yōko, le mariage doit être le lieu qui permet la réalisation de la personnalité de chacun des conjoints, grâce à ce qu'elle appelle « l'amour sacré ". Comme les autres contributrices, elle interroge le sens que le mariage peut avoir pour la femme, et insiste sur la nécessité d'acquérir une individualité authentique, indépendamment du rôle d'épouse et de mère : "Nora aurait dû accéder à un sens plus profond de sa vie. Elle aurait dû savoir ce que signifie une vie en adéquation avec son moi authentique. " (Horiba 1991 : 36).

Elle fait preuve de modération en abordant l'avenir de Nora : elle défend la nécessité de revenir au foyer une fois qu'elle aura construit sa personnalité. Si la femme doit devenir un individu moderne, pour Yōko, elle ne doit 
pas tomber dans l'individualisme qu'elle qualifie d'infantile : « tout sacrifier au nom d'un but individuel relève d'une subjectivité infantile" (ibid. : 42). Même si elle critique le confinement de la femme au foyer qui entraîne chez cette dernière des tendances conservatrices, voire réactionnaires, contraires à l'émancipation féminine, le couple idéal reste pour elle celui où la femme se consacre à son mari et à sa carrière :

«Elle doit devenir l'interlocutrice privilégiée de l'homme avec qui elle pourra prendre des décisions et même devenir le bras droit de sa réussite. [...] Elle deviendra ainsi, sans rancœur aucune, la compagne de sa vie et remplira son rôle d'assistante avec fierté. » (Ibid: 43).

La nécessité du retour au foyer est aussi évoquée dans le texte de Yasumochi Yoshi qui avait joué un rôle essentiel en encourageant Raichō à créer l'association et la revue Seitō.

Ueno Yō est à la fois plus modérée et plus optimiste que Raichō : cette dernière estime que l'homme est égoïste par nature dans sa relation aux femmes, et critique la naïveté de Nora qui croyait le contraire, tout en lui concédant la difficulté accrue qu'il y a à démasquer chez l'homme occidental civilisé ce caractère égoïste : " la seule différence entre eux consiste dans l'habileté à le dissimuler, et la distinction entre civilisé et barbare ne réside que dans cette différence " (Horiba 1991 : 64). Par ces expressions, Raichō semble prendre ses distances par rapport à l'opposition civilisé/barbare mise en avant par Fukuzawa Yukichi 福澤諭吉 (1834-1901) dans son Bunmei gairyakuron 文明概略論 (Bref traité sur la civilisation, 1875) ${ }^{38}$, alors qu'Ueno Yōko, en recourant à la métaphore entre le régime despotique et le régime constitutionnel dans la relation du couple, reste dans la perspective tracée par celui-ci. D’ailleurs, dès la première phrase, Raichō se montre critique envers Nora dont elle oppose la naïveté à la maturité que toute Japonaise dans sa situation aurait déjà acquise :

"Chère Nora, une femme comme vous qui n'obéit qu’à son instinct, et aussi aveugle, on peut se l'imaginer à condition qu'elle soit une jeune fille de quatorze ou

38. Pour Fukuzawa, les pays occidentaux sont civilisés, les pays d'Asie et d'Afrique barbares, à l'exception du Japon qui est semi-développé. L'urgence pour le Japon est de rejoindre les pays civilisés et, en 1894-95, il qualifie la guerre sino-japonaise de croisade de la civilisation contre la Chine "barbare". 
quinze ans, mais aux yeux des Japonaises, que vous soyez la mère de trois enfants, cela paraît incroyable." (Horiba 1991 : 62).

Raichō est la seule à ne pas se préoccuper du couple en particulier, questionnant exclusivement l'éveil, la vraie prise de conscience, le long cheminement qui l'attend encore avant la découverte de son propre moi, les difficultés qu'il y a à se débarrasser des illusions.

L'hésitation entre la modération et la hardiesse frappe le lecteur d'aujourd'hui. Ueno Yōko, si elle insiste pour que l'émancipation puisse se préparer en douceur, et que l'homme n'ait plus d'autre choix que de "s'incliner devant elle ", réaffirme aussitôt après la nécessité de la rupture, même violente, dans le cas où la femme est transformée en un instrument au service de l'homme :

"Alors elle prend conscience de ce que représente l'épouse pour le mari : elle n'est au fond qu'un instrument à son service ! Sa personnalité est sous son joug. Par bonheur, Nora n'avait pas perdu son instinct inné de femme. Femme, portant sur ses épaules le destin de femme, celle qui représente la moitié de l'humanité, Nora se décide à se battre, et pense que pour réaliser cette immense tâche, elle doit, même au sacrifice de son attachement, préserver sa dignité de femme. "

Et, elle ajoute :

"Dans ce sens, on peut même parler de chant victorieux de la femme, même s'il ressemble à une tragédie. [...] Une si grande révolution - la grande révolution entre les sexes - n'est pas aisée à réussir. " (Horiba 1991: 43).

Mais elles veulent toutes garder la perspective de créer un foyer heureux, c'est-à-dire celui où la valeur de chacun est reconnue. Cet optimisme contraste avec les interprétations ultérieures ou avec les suites pessimistes envisagées par Lu Xun (1981 : 179-185), Mori Ōgai'39, Elfried Jelinek (1993) ou encore le metteur en scène Ostermeier ${ }^{40}$.

39. Son roman Gan 雁, Les Oies sauvages (1911-1913), est souvent cité comme un exemple de pessimisme quant aux perspectives possibles de l'émancipation féminine dans la société japonaise.

40. Cette mise en scène a suscité la polémique : Nora, à la fin de la pièce, tue son mari, ce qui pour Ostermeier serait la seule issue possible pour la Nora moderne. Pour ses détracteurs, cette Nora n'a rien à voir avec celle d'Ibsen. Le metteur en scène aura au 
Elle ne repose pas tant sur l'idéalisation du couple que sur l'idée de l'élévation du statut de la femme dans la société moderne. Pour ce faire, elle énumère les principales revendications : l'obtention du droit de participer à la vie politique, la révision de la loi sur l'adultère, la possibilité pour les femmes de rester célibataires avec les mêmes droits que les hommes. Son texte garde un ton très optimiste jusqu'à la fin où elle appelle à l'émancipation de la femme en Occident et en Orient. L'affirmation du caractère universel du message de la pièce est très présente également dans l'article écrit par Ueda Kimi. Celle-ci est déjà une auteure reconnue qui a reçu le premier prix en 1908 pour son roman Kurobotan 黒牡丹 (Pivoine noir) au concours de nouvelles organisé par le quotidien Ōsaka Mainichi shinbun 大阪毎日 新聞. Elle a une approche très directe, résumant la pièce au drame auquel conduit le conflit intérieur qui traverse la femme moderne, et comparant la situation de Nora à un état d'esclavage. Comme Ueno Yōko, elle souligne le caractère universel du combat des femmes : "Ce cri rempli de tristesse, c'est le cri de toutes les Scandinaves. Non, c'est la plainte des femmes du monde entier ». Sa sympathie pour Nora est sans ambiguïté :

« Nous qui nous éveillons, [...] nous qui sommes tourmentées en permanence par la vieille morale, par les anciennes coutumes, une fierté et une joie sans borne nous envahissent mystérieusement pour nous faire crier victoire quand Nora s'en va. " (Horiba 1991 : 60).

Nora agit aussi à leur place et leur donne du courage.

Ce sont les mêmes idées qui sont développées chez les autres auteurs : l'idée que le degré d'émancipation de la femme est un indice du degré d'avancement de la société, que la réalisation de la vraie personnalité est la condition au vrai mariage - celui de l'amour réciproque et de l'égalité opposé au mariage conventionnel.

moins posé une question : le mouvement de libération de la femme a-t-il réussi, peut-il réussir? 


\section{La réaction de Hiratsuka Raichō}

Hiratsuka Raichō fait figure d'exception, et si nous ne pouvons pas ici examiner toutes les raisons possibles, une des clés se trouve dans la publication du roman que Morita Sōhei 森田草平 (1881-1949) avait tiré de l'affaire connue sous le nom de Shiobara jiken 塩原事件 $(1908)^{41}$. Dans ce roman, Baien 煤煙 (Suie), écrit en 1909, et dont le titre fut directement inspiré, d'après Raichō, de Fumée de Tourgéniev qu'il lui avait donné à lire dans une version anglaise, il compare l'héroïne, Tomoko (Raichō pour tout lecteur de l'époque), à Nora, mais plus sûre d'elle. Les membres de Seitō comme Otake Kōkichi (Beniyoshi), Araki Ikuko 荒木郁子 (1888-1943) et Ikuta Hanayo avaient lu Baien et avaient été très attirées par Raichō en qui elles avaient vu précisément l'héroïne du roman. Raichō voulait probablement se démarquer de Nora, ou de la représentation que les lectrices ou lecteurs en avaient eue.

Une Maison de poupée faisait partie des lectures qu’affectionnait Morita. Raichō, passablement énervée par le roman que venait de publier Morita qui révélait au public les aspects intimes de l'affaire et de plus faisait durer le scandale à son détriment (Odaira 2008 : 159-167), avait peut-être le désir de « surpasser» Nora?

De cette affaire, dans ses mémoires, elle affirme sa déception, et garde le sentiment d'avoir servi l'écrivain dans sa recherche abstraite et littéraire du sens du mot ren.ai 恋愛, l'amour romantique. Elle même à l'époque en avait ressenti une "dépression de la personnalité " (Horiba 1991 : 16) qui eut en même temps la fonction de lui révéler son identité sexuée.

Dans son commentaire sur Nora, prenant la forme d'une lettre qui lui était directement adressée, donc de l'apostrophe, elle affirmait sa propre personnalité acquise grâce à son entraînement assidu à la méditation au sein d'un temple de la secte bouddhiste zen Rinzai (Rinzai shū 臨済宗).

La conception bouddhiste de la nature du moi se rattache à celle du "nirvana ( nehan 涅槃 en japonais) qui exige l'extinction des passions, des désirs, des illusions, et donc - in fine - des attachements de toute sorte, à commencer par le premier d'entre eux : l'attachement à un moi autonome

41. C'est une histoire de fugue dont le but avait été le double suicide (shinjū 心中 en japonais) entre Raichō et cet écrivain qui renonça une fois sur place à cette idée. 
et permanent. Cette conception plaçait Raichō dans une approche complexe, la prise de conscience de la vraie nature, totalement libérée, du moi, impliquant un parcours qui pouvait être d'une rigueur extrême (ascèse érémitique), au terme duquel se produirait ou non l'Éveil.

La phrase suivante qu'elle lui adresse reflète bien cette conception du moi qui résulte de la foi religieuse qu'elle garda toute sa vie :

" "Qu'est-ce que mon moi total", voilà la question qui vous préoccupera. Que découvrirez-vous ? Que ce que vous preniez pour vous-même jusqu'à présent n’est que chimère, illusion, et que ce fantôme jusqu'à présent ne faisait que cacher votre vrai moi.

"Nora, voilà votre deuxième tragédie qui vous attend. La dissolution de cette chimère, de cette illusion, la négation de soi, le dur combat pour arriver à l'anéantissement de soi. » (Horiba 1991 : 66).

\section{Conclusion}

Dans ces textes, la question de la valorisation personnelle de la femme au sein du couple grâce à l'établissement d'un nouveau modèle de mariage - le mariage d'amour - constitue l'essentiel de la préoccupation des membres de Seitō. Ce souci est corrélatif de la formation du sujet abstrait à partir de l'individu, qui n'est plus défini seulement par ses rôles sociaux, et explique la place accordée à la question de la personnalité intérieure. La position de Raichō différait de celle des autres, parce que sa conception de l'individu et de la personnalité intérieure était moins tributaire de l'expérience amoureuse que de la concentration spirituelle ou religieuse, conception qui évolua chez elle après sa découverte d'Ellen Key.

Les débats autour des pièces de théâtre ne se réduisirent pas à celui portant sur le personnage de Nora dans la revue Seitō ; d'autres héroïnes, comme Hedda Gabler et, deux ans plus tard, Magda ${ }^{42}$ firent l'objet de discussions passionnées. Mais le débat sur Nora prépara celui sur la femme nouvelle que la revue allait entreprendre un an plus tard.

42. La pièce de Sudermann (Heimat 1893, traduite en anglais sous le titre Magda) fut présentée au Japon sous le titre Kokyō 故郷 (Pays natal) en mai 1912, mais la fin fut censurée pour atteinte à l'ordre moral. 
Le succès de la pièce et les débats autour du personnage de Nora offrirent un moment privilégié de l'histoire des femmes et du théâtre : les membres de Seitō n'eurent pas de positions unifiées, mais elles insistaient toutes sur la question de la prise de conscience de la femme en tant qu'individu et dénonçaient les coutumes et les mentalités qui l'en empêchaient. De ce point de vue, leurs positions, bien que modérées ${ }^{43}$, par leur volonté de mettre au premier plan la femme en tant qu'individu, diffèrent radicalement des points de vue affirmés par l'ensemble des commentateurs ou des éducateurs de leur époque. Le contexte politique et idéologique de l'époque rendait toute action politique directe impossible : le numéro de février 1913, qui comprend un article de Fukuda Hideko 福田英子 (1865-1927) sur les perspectives politiques de l'émancipation de la femme, sera interdit aussitôt. Chez beaucoup pointe l'idée de la difficulté de réussir à la fois l'émancipation féminine et le mariage d'amour ou la relation amoureuse : la question de la reconnaissance du célibat comme mode de vie est posée. Elles n'opposent pas réussite sociale et réussite amoureuse, loin de là, mais semblent penser néanmoins que l'obstacle principal, dû à l'incompréhension de l'homme vis-à-vis des aspirations féminines, les empêche souvent d'atteindre les deux buts. Et plutôt que de se soumettre et de se résigner à vivre un mariage sans amour, c'est-à-dire sans reconnaissance de leur propre valeur, elles pensent qu'il vaut mieux choisir la solitude, le célibat.

La grande question à venir reste celle de la définition de cette valeur et des modalités de sa reconnaissance dans la société. Chacune des protagonistes de ces débats prendra part à des courants idéologiques et politiques divers dans les années ultérieures, en particulier au lendemain de la Première Guerre mondiale, mais le féminisme de cette période a posé pour la première fois au Japon la question du lien entre l'émancipation de la femme dans la sphère privée de la famille et sa reconnaissance sociale, en exposant publiquement les diverses préoccupations intérieures et personnelles des femmes, rejoignant ainsi la grande thématique du dépassement de la frontière entre le privé et le public du féminisme de la seconde vague des années 1960 et 1970.

43. Les membres de Seitō ne cherchèrent pas à donner un caractère politique à leurs revendications ni à lancer immédiatement une campagne pour un changement juridique de la position de la femme mariée. 


\section{Bibliographie}

\section{BARDSLEY Jan 2007}

The Bluestockings of Japan:

New Woman Essays and Fiction from

Seitō, 1911-16, Center for Japanese

Studies, Ann Arbor, The University

of Michigan.

\section{BENHAMOU Anne-Françoise (dir.) \\ 2011 \\ "Contemporaines ? Rôles féminins dans le théâtre d'aujourd'hui », \\ OutreScène, juin, $\mathrm{n}^{\circ} 12$, \\ La Colline-Théâtre national.}

\section{BRAUNSCHWEIG Stéphane 2003}

"Une clarté qui n'éclaire pas », in Ibsen, OutreScène, revue du Théâtre national de Strasbourg, mars, 2 : 53-60.

BRAUNSCHWEIG Stéphane 2009/2010 Rosmersholm/Une Maison de poupée, mise en scène $S$. Braunschweig, La Colline-Théâtre national.

\section{COLLETT Camilla 2010}

Les Filles du préfet, traduit du norvégien par Eric Eydoux, Carouge, Éditions Zoe.

\section{FAALAND Maryline 2009}

«Aspects du génie littéraire norvégien: aux sources du sentiment identitaire. De Wergeland à Olav Duun ", thèse sous la direction de Marc Auchet, université Paris-Sorbonne.

\section{FUKATANI Masashi 深谷昌志} [1981] 1998

Ryōsai kenbo shugi no kyōiku 良妻賢母 主義の教育 (L'éducation selon l'idéologie de la « bonne épouse, mère avisée »), Nagoya, Reimei shobō 黎明書房.

\section{GRAVIER Maurice 1968}

Le féminisme et l'amour dans la littérature norvégienne, 1850-1950, Paris, Minard.

\section{HAGA Noboru 芳賀登 1990}

Ryōsai kenboron 良妻賢母論 (Théorie sur la bonne épouse, mère avisée), Tokyo, Yūzankaku shuppan 雄山閣出版.

HIRATSUKA Raichō 平塚らいてう 1992 Genshi, josei wa taiyō de atta 元始、女性は太陽であった (À l'origine, la femme était le soleil), Tokyo, Ōtsuki shoten 大月書店, coll. Kokumin bunko

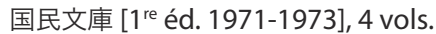

\section{HIRATSUKA Raichō 2006}

In the Beginning, Woman Was the Sun, traduit et annoté par CRAIG Teruko, New-York, Columbia University Press.

\section{HORIBA Kiyoko 堀場清子 1991}

Seitō josei kaihō ronshū

『青鞮』女性解放論集 (Recueil de textes sur la libération de la femme dans Seitō), Tokyo, Iwanami shoten 岩波書店.

IIDA Yūko 飯田祐子 (dir.) 2002

Seitō to iu ba. Bungaku, jendā, 《atarashii onna »『青鞜』という場

一文学・ジェンダー・〈新しい女〉

(Seitō comme lieu. Littérature, genre, «femme nouvelle »), Tokyo, Shinwasha 森話社. 
JELINEK Elfried 1993

Ce qui arriva quand Nora quitta son mari, Paris, L'Arche.

\section{Joseishi sōgō kenkyūkai}

女性史総合研究会 1982

Nihon joseishi 日本女性史, vol. 4 Kindai

近代 (L'époque moderne), vol. 5 Gendai

現代 (L'époque contemporaine),

Tokyo, Tokyo daigaku shuppankai

東京大学出版会.

\section{KANO Ayako 2001}

Acting Like a Woman in Modern Japan, New-York, Palgrave.

KAWAMURA Karyō 川村花菱 [1968] 2006

Matsui Sumako : Geijutsuza seisuiki 松井須磨子一一芸術座盛衰記 (Matsui

Sumako : prospérité et décadence du Geijutsuza), Tokyo, Seiabō 青蛙房.

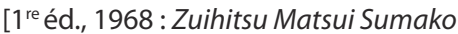
随筆 松井須磨子 (Essai sur Matsui Sumako)].

KŌCHI Nobuko 香内信子 (éd.) 1984 Shiryō bosei hogo ronsō 資料 母性保護 論争 (Documents : polémiques sur la protection de la maternité), Tokyo, Domesu shuppan ドメス出版.

\section{KOTT Jan 2003}

"Ibsen, une relecture », in Ibsen, OutreScène, revue du Théâtre national de Strasbourg, $2: 61$ - 94.

\section{KUWAKI Gen.yoku 桑木厳翼 1906}

《Kinsei gikyoku to jinsei » 近世戯曲と 人生 (Le théâtre moderne et la vie), in Jidai shichō 時代思潮 (Courants de pensée contemporains), février.

\section{LÉVY Christine 2010}

«Autour de l'Affaire de crime du lèsemajesté : modernité politique et répression », Ebisu-Études japonaises, «La modernisation du Japon revisitée. Que reste-t-il de l'approche moderniste ? », 44 : 87-109.

\section{LOWY Dina 2007}

The Japanese "New Woman". Images of Gender and Modernity, New Brunswick, N. J., Rutgers University Press.

\section{LU Xun 1981}

"Qu'est-il advenu de Nora après son départ ? ", in La Tombe, Paris, Acropole/ UNESCO.

\section{MILL John Stuart 密爾 1878}

Danjo dōkenron 男女同権論 (Théorie sur l'égalité des droits entre hommes et femmes), traduction de The Subjection of Women par Fukamauchi Motoi 深間内基, Tokyo, éd. Yamanaka Ichibei 山中市兵衛.

MORITA Sōhei 森田草平 1995 [rééd.] Baien 煤煙 (Suie), Tokyo, Iwanami shoten 岩波書店.

MUTA Kazue 牟田和惠 2006

Jendā kazoku o koete ジェンダー家族を 超えて(Dépasser la famille basée sur le genre), Tokyo, Shin.yōsha 新曜社.

NAKAMURA Toshiko 中村都史子 1997 Nihon no Ipusen genshō: 1906-1916 nen 日本のイプセン現象—1906-1916年 (Le phénomène Ibsen au Japon, années 
1906-1916), Fukuoka, Kyūshū daigaku shuppankai 九州大学出版会.

\section{Nihon bungaku kyōkai \\ Shin-feminizumu hihyō no kai \\ 日本文学協会 新・フェミニズム批評の会 \\ (Association pour une nouvelle critique féministe) (éd.) 1998 \\ Seitō o yomu『青鞜』を読む (Lire Seitō), Tokyo, Gakugei shorin 學藝書林.}

ODAIRA Maiko 小平麻衣子 2008

Onna ga onna o enjiru: bungaku, yokubō, shōhi 女が女を演じる—文学・欲望·消費 (La femme dans le rôle de la femme: littérature, désir et consommation), Tokyo, Shin.yōsha 新曜社.

\section{ORII Miyako 折井美耶子 1991}

Shiryō sei to ai o meguru ronsō 資料性と 愛をめぐる論争 (Documents : autour des polémiques sur les questions de sexualité et d'amour), Tokyo, Domesu shuppan ドメス出版.

\section{OSTERMEIER Thomas 2003}

«Un regard matérialiste sur le présent, entretien autour de Maison de Poupée ", in Ibsen, OutreScène, revue du Théâtre national de Strasbourg, 2 : 45-52.

\section{RODD Laurel Rasplica 1991}

"Yosano Akiko and the Taishō Debate Over the "New Woman"», in BERNSTEIN Gail Lee (éd.), Recreating Japanese Women, 1600-1945, Berkeley, University of California Press.

SASAKI Hideaki 佐々木英昭 1994 Atarashii onna no tōrai. Hiratsuka Raichō to Sōseki 新しい女の到来——平塚らい
てうと漱石 (L'apparition de la femme nouvelle. Hiratsuka Raichō et Sōseki), Nagoya, Nagoya daigaku shuppankai 名古屋大学出版界.

\section{SASAKI Hideaki 佐々木英昭 \& NEGISHI Masazumi 根岸正純 1999 Baien : shōchū 煤煙——詳注 (Suie : commentaires et analyses), Kyōto, Kokusai nihon bunka kenkyū sentā 国際日本文化研究センター.}

\section{Seitōsha 青鞜社 1911-1916} Seitō. Fukkoku-ban 青鞜復刻版 (Seitō. Fac-similé), Tokyo, Ryūkei shosha 龍渓書舎, 1980 ; Fuji shuppan 不二出版, 1983.

TANAKA Yūkichi 田中祐吉 2005 《Josei no dokushin-nan » 女性の独 身難 (Les difficultés du célibat chez les femmes), Joshi bundan 女子文壇 décembre 1912, édition fac-similé, Tokyo, Fuji shuppan 不二出版.

\section{TOMIDA Hiroko 2004}

Hiratsuka Raicho and Early Japanese Feminism, Leiden, Boston, Brill.

Tsubouchi kyōkai 逍遥協会 1998 Tsubouchi Shōyō kenkyū shiryō, dai jūroku shū 坪内逍遥研究資料第16集 (Études sur Tsubouchi Shōyō, 16 recueil), Tokyo, Shinjusha 新樹社.

TSUBOUCHI Shōyō 坪内逍遥 1912 Iwayuru atarashii onna 所謂新シイ女 (La femme nouvelle en question), Tokyo, Seibidō 精美堂. 
YONEDA Sayoko 米田佐代子 2002

Hiratsuka Raichō. Kindai Nihon no demokurashi to jendā 平塚らいてう 近代日本のデモクラシーとジェンダー (Hiratsuka Raichō. La démocratie et le genre dans le Japon moderne), Tokyo, Yoshikawa kōbunkan 吉川弘文館. 